\title{
The correlation between cell-free DNA and tumour burden was estimated by PET/CT in patients with advanced NSCLC
}

\author{
A D Nygaard ${ }^{\star}, 1$, P C Holdgaard ${ }^{2}$, K-L G Spindler ${ }^{1}$, N Pallisgaard ${ }^{3}$ and A Jakobsen ${ }^{1}$ \\ ${ }^{1}$ Department of Oncology, Vejle Hospital, Kabbeltoft 25, 7100 Vejle, Denmark; ${ }^{2}$ Department of Nuclear Medicine, Vejle Hospital, \\ Kabbeltoft 25, 7100 Vejle, Denmark and ${ }^{3}$ Department of Molecular Biochemistry, Vejle Hospital, Kabbeltoft 25, 7100 Vejle, \\ Denmark
}

Background: Cell-free DNA (cfDNA) circulating in the blood holds a possible prognostic value in malignant diseases. Under malignant conditions, the level of cfDNA increases but the biological mechanism remains to be fully understood. We aimed to examine the correlation between cfDNA and total tumour burden defined by positron emission tomography (PET) parameters.

Methods: Patients with advanced non-small cell lung cancer (NSCLC) were enrolled into a prospective biomarker trial. Before treatment, plasma was extracted and the level of cfDNA was determined by qPCR. An ${ }^{18} \mathrm{~F}$-fluorodeoxyglucose $\left({ }^{18} \mathrm{~F}-\mathrm{FDG}\right)$ PET/computed tomography (CT) scan was performed and evaluated in terms of metabolic tumour volume (MTV) and total lesion glycolysis (TLG). Tumour contours were delineated semi-automatically by a threshold standardised uptake value (SUV) of 2.5 . The primary end point was correlation among cfDNA, MTV and TLG. The secondary end point was overall survival (OS) according to cfDNA, MTV and TLG.

Results: Fifty-three patients were included. There were no correlations between cfDNA and MTV $(r=0.1)$ or TLG $(r=0.1)$. cfDNA $>75$ th percentile was correlated with shorter OS $(P=0.02)$, confirmed in a multivariate analysis. MTV $>$ the median was associated with a significantly shorter OS $(P=0.02)$. There was no significant difference in OS according to TLG $(P=0.08)$.

Conclusion: Cell-free DNA may not be a simple measure of tumour burden, but seems to reflect more complex mechanisms of tumour biology, making it attractive as an independent prognostic marker.

Lung cancer is one of the most common cancers worldwide and associated with considerable morbidity and mortality. The choice of treatment is based on the extent of disease at the time of diagnosis. In non-small cell lung cancer (NSCLC), patients with localised disease may be offered curatively intended surgery or chemo-radiotherapy, whereas patients with advanced disease are treated with chemotherapy and/or biological drugs in a palliative setting (Rinaldi et al, 2006; Gorenstein and Sonett, 2011; Mok et al, 2012). The treatment is often associated with side effects and the long-term survival is poor. During recent years, individualised treatment strategies have gained an increasing interest. The possibility of targeting the treatment and thereby avoiding unnecessary toxicity and hopefully increasing the treatment response is attractive, but it requires mapping of the tumour biology. One way of gaining further information about the tumour is by using tumour markers. Many different markers have been and are currently investigated, but so far only few have proved to be clinically useful (Vadakara and Borghaei, 2012).

One potential marker is cell-free DNA (cfDNA), which are small fragments of DNA detectable in the blood (Leon et al, 1977). Several studies suggest that cfDNA is shed by both normal cells and tumour cells, making it potentially attractive as an easily accessible source of information on tumour biology. During malignant conditions, the level of cfDNA is believed to increase 
due to increased release from the tumour cells, and possibly also from normal cells in the tumour surrounding tissue. The biological mechanisms behind the increased release remain to be fully understood, but elements of apoptosis, necrosis, especially of large tumours, and the possibility of active release from the tumour have been presented (Stroun et al, 2000). Several studies have demonstrated a prognostic value of the level of cfDNA in different malignancies (De et al, 2011; De Mattos-Arruda et al, 2011; Ellinger et al, 2011; Punnoose et al, 2012; Schwarzenbach et al, 2011; Spindler et al, 2012; Dawson et al, 2013). These observations have led to the question of whether the increased level of cfDNA merely represents an increased tumour burden, or whether other biological mechanisms associated with the aggressiveness of the tumour are involved. It seems to be plausible that more aggressive tumours cause higher levels of cfDNA, thereby impairing the prognosis, but further investigations addressing this issue are needed.

During the disease staging process, many of the patients with NSCLC have functional imaging performed with positron emission tomography/computed tomography (PET/CT), using ${ }^{18} \mathrm{~F}$-fluorodeoxyglucose $\left({ }^{18} \mathrm{~F}-\mathrm{FDG}\right)$, which enables estimation of volumebased parameters such as the metabolic tumour volume (MTV) and the total lesion glycolysis (TLG) (Gould et al, 2001). The first estimate (MTV) is a purely volumetric entity, while TLG also takes the metabolic activity of the lesion into account, thereby providing an estimate of the tumour activity. Hence, PET parameters provide a means of estimating the tumour burden and also, to some extent, the metabolic activity and aggressiveness of the disease. A prognostic value of either whole-body or primary tumour MTV and TLG in NSCLC has previously been demonstrated with higher values correlating with shorter progression-free survival (PFS) and overall survival (OS) (Lee et al, 2007; Chen et al, 2012; Kim et al, 2012; Liao et al, 2012; Zaizen et al, 2012; Zhang et al, 2012, 2013; Hyun et al, 2013).

The aim of our study was to investigate the correlation between the baseline level of cfDNA and volumetric parameters of the $\mathrm{PET} / \mathrm{CT}$ scan, and to evaluate the prognostic value of cfDNA and the PET parameters.

\section{MATERIALS AND METHODS}

Patients. During a 3-year period (2007-2010), patients with newly diagnosed advanced NSCLC referred for first-line chemotherapy were enrolled into a prospective biomarker trial at the Department of Oncology, Vejle Hospital, Denmark as previously presented (Nygaard, 2012). The inclusion criteria comprised histopathologically confirmed NSCLC stage III or IV, no previous chemotherapy, performance status (PS) $\leqslant 2$ and age above 18 years. All patients had a pre-treatment peripheral blood sample drawn from which plasma was isolated and stored at $-80^{\circ} \mathrm{C}$. Patients were subsequently treated with standard first-line chemotherapy comprising carboplatin (dose based on AUC5, given i.v. on day 1 every 3 weeks) in combination with vinorelbine $\left(30 \mathrm{mg} \mathrm{m}^{-2}\right.$ given i.v. on day 1 every 3 weeks and $60 \mathrm{mg} \mathrm{m}^{-2}$ p.o. day 8 every 3 weeks) for a maximum of six cycles. One patient was additionally treated with bevacizumab in combination with the chemotherapy. Palliative radiotherapy was given if indicated.

When indicated, the routine staging was supplemented by a PET-CT scan. The PET scan was evaluated in terms of whole-body MTV and TLG by a dedicated nuclear medicine specialist, blinded to the level of cfDNA. Only patients with a PET/CT scan within a month before blood sampling were included in the present study.

All patients had a baseline CT of the chest and upper abdomen within a month before treatment start. Control CT scans were performed after every two treatment cycles and every 3 months after finishing chemotherapy, or earlier when indicated. Objective response was evaluated according to RECIST v.1.0 (Therasse et al, 2000). The primary end point was correlation between the baseline level of cfDNA and tumour burden estimated by whole-body MTV and TLG. The secondary end points were PFS and OS according to the level of MTV, TLG and cfDNA.

Written informed consent was obtained before enrolment. The study was approved by the Regional Scientific Ethical Committee for Southern Denmark in accordance with Danish law.

The REMARK criteria were considered and applied whenever possible (McShane et al, 2005).

Analysis of cfDNA. Plasma was obtained from a pre-treatment peripheral $10 \mathrm{ml}$ EDTA-blood sample by centrifugation at $2000 \mathrm{~g}$ for $10 \mathrm{~min}$, and stored at $-80^{\circ} \mathrm{C}$. The plasma was prepared within $2 \mathrm{~h}$ after sampling. Total nucleic acid was obtained from $1.0 \mathrm{ml}$ plasma by the use of a QIAsymphony virus/bacteria midi-kit on a QIAsymphony robot (Qiagen, Hilden, Germany) according to the manufacturer's instructions. Analyses of total number of cfDNA alleles were performed by an in-house developed method targeting the peptidylprolyl isomerase A (PPIA) gene as described and validated previously (Steffensen et al, 2011; Spindler et al, 2012).

PET/CT imaging and image analysis. The PET scans were performed using a GEMINI TF/16 channel PET/CT scanner (Philips Medical Systems, Cleveland, OH, USA). An average of $310 \mathrm{MBq}(271-405 \mathrm{MBq})$ of ${ }^{18} \mathrm{~F}-\mathrm{FDG}$ was injected intravenously. Images were obtained $1 \mathrm{~h}$ after injection of the tracer covering from the base of the skull to the upper thigh with 2 min per bed position. The patient preparation was in accordance with the EANM procedure guideline (Boellaard et al, 2010). Images were reconstructed with the Philips reconstruction protocol: BodyCTAC-NAC (LOR-based OSEM with TOF; iterations: 3; subsets: 33). Images were analysed on a Philips Extended Brilliance Workspace (Philips Healthcare, Best, The Netherlands), v. 4.5.3.40140 using the Tumour Tracking software. All scans were attenuation corrected with a low-dose CT ( $50 \mathrm{mAs}, 120 \mathrm{kV}$, slice thickness $5 \mathrm{~mm}$, increment $2.5 \mathrm{~mm}$, pitch 0.81 , collimation $16 \times 1.5 \mathrm{~mm}$ ). The patient's baseline, iv contrast enhanced, high-dose CT images were also used when evaluating the PET/CT. The ${ }^{18}$ F-FDG uptake was assessed using the standardised uptake value (SUV), which normalises the radioactivity measured in tissue by the injected dose and the body weight of the patient. Threedimensional volumes of interest (VOIs) were calculated automatically for all tumour lesions using the 'bounded brick' tool with a threshold of $2.5 \mathrm{SUV}$ including primary tumour, lymph nodes and metastases. If the VOIs included physiological FDG uptake, then they were manually corrected using the 'interactive' tool. Volumes of interest were created by an experienced nuclear medicine PET/CT specialist blinded to the level of cfDNA. Each patient had a MTV calculated as the sum of each VOI. For each lesion volume and SUV mean was multiplied and then summed to generate the TLG.

Statistics. Correlations between patient characteristics and cfDNA, MTV and TLG were analysed by the Wilcoxon ranksum test. The correlation between the level of cfDNA, MTV and TLG was determined by the non-parametric Spearman's rank correlation method. Survival data were analysed by the KaplanMeier method, and differences between the groups were estimated by the log-rank test.

Objective response was determined according to the RECIST 1.0 criteria.

Progression-free survival was defined as time elapsed between the first day of treatment and date of progression or death from any cause. Overall survival was defined as time between the first day of treatment and date of death from any cause. Patients who did not experience any event were censored by 1 November 2012. 
The independent effect of different variables was investigated in multivariate Cox regression analysis. Known prognostic variables and the variables of interest in the study (cfDNA, MTV and TLG) were included in the model.

All statistics were carried out in the NCSS statistical software (NCSS Statistical Software, Kaysville, UT, USA; version 07.1.15, 2009). Two-sided $P$-values $<0.05$ were considered as significant.

\section{RESULTS}

Patients. Fifty-three patients fulfilled the criterion of a PET/CT scan within a month (median 13 days) before the blood sampling and were included in the study. Patient characteristics are listed in Table 1. The level of cfDNA, whole-body MTV and TLG was determined in all patients. The level of cfDNA ranged from 450 to 133800 alleles per $\mathrm{ml}$ with a median of 2200 . The median wholebody MTV was $95 \mathrm{ml}$ (range 3-647) and the median TLG was 444 (range 12-4435).

The correlation between patient characteristics and cfDNA and PET parameters was investigated and revealed a significantly lower TLG in patients with adenocarcinomas compared with patients with squamous cell carcinoma. There were no other significant correlations.

Correlation between MTV, TLG and cfDNA. As demonstrated in Figure 1, there was no correlation between the level of cfDNA and MTV (correlation coefficient $r=0.1$ ). Likewise, no correlation between the level of cfDNA and TLG was detected ( $r=0.1)$. On the

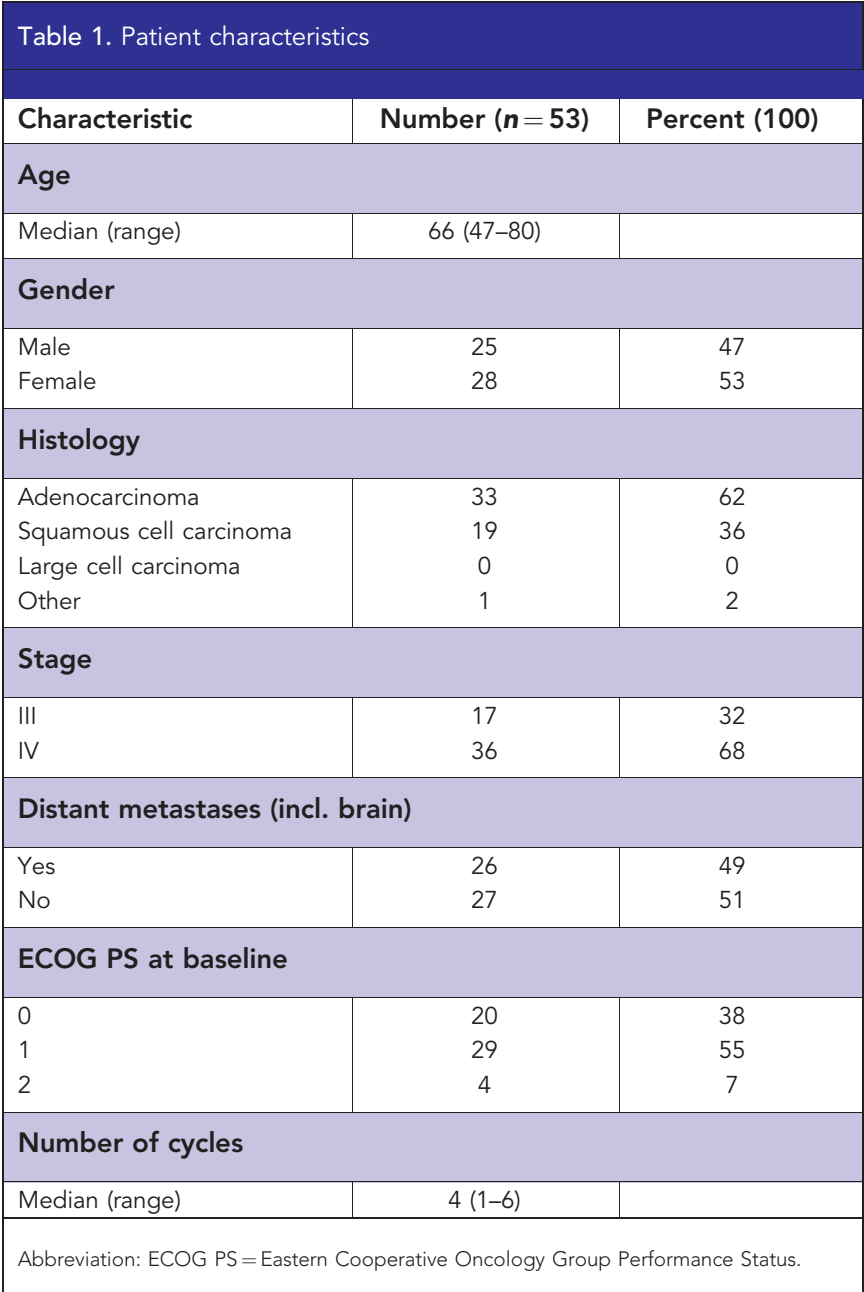

other hand, there was an almost perfect correlation between MTV and TLG $(r=0.97)$.

Survival analyses. The median PFS was 5.9 months (95\% CI 5.6-7.1) and the median OS was 15.7 months (95\% CI 10.8-21.8). Survival data were further analysed according to the levels of cfDNA, MTV and TLG. To determine the most appropriate cutoff, all of the three parameters were divided into quartiles. This exploratory approach revealed a significantly shorter OS of patients with cfDNA in the upper fourth quartile compared with the lowest quartile. There were no significant differences in MTV and TLG according to any of the quartiles. Consequently, we dichotomised cfDNA by the 75th percentile, and MTV and TLG by the median.

As demonstrated in Figure 2, the median OS of patients with cfDNA below the 75th percentile was 17.8 months (95\% CI 13.0-24.5) compared with 8.8 months (95\% CI 6.7-11.5) in patients with cfDNA levels above the 75th percentile. The hazard ratio (HR) was 2.05 (95\% CI $0.94-4.50)$ with a $P$-value of 0.02 . Furthermore, high MTV was also associated with a shorter OS. The OS of patients with MTV below the median was 21.9 months (95\% CI 13.0-26.9) compared with 7.0 months (95\% CI 5.9-16.8) in patients with MTV above the median (HR: 1.87 (95\% CI 1.04-3.38), $P=0.02)$. There were no significant differences according to the level of TLG $(P=0.08)$. The same applied to PFS, with no significant differences according to any of the parameters (data not shown).

Multivariate analysis. To investigate the independent prognostic value of cfDNA and the PET parameters, multivariate Cox regression analyses of OS were performed. Due to the low number of events, only five parameters were included in the analyses. The model is demonstrated in Table 2. In addition to cfDNA,
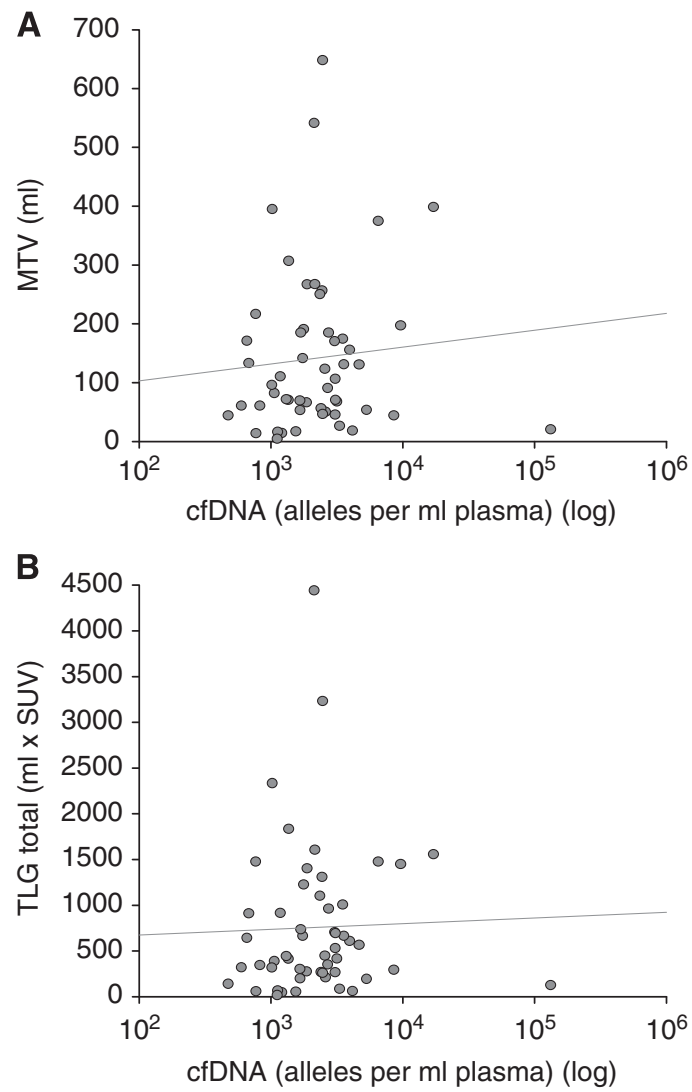

Figure 1. Correlation between the level of cfDNA and PET parameters. (A) cfDNA and MTV: correlation coefficient $(r)=0.1$. (B) cfDNA and TLG: correlation coefficient $(r)=0.1$. 

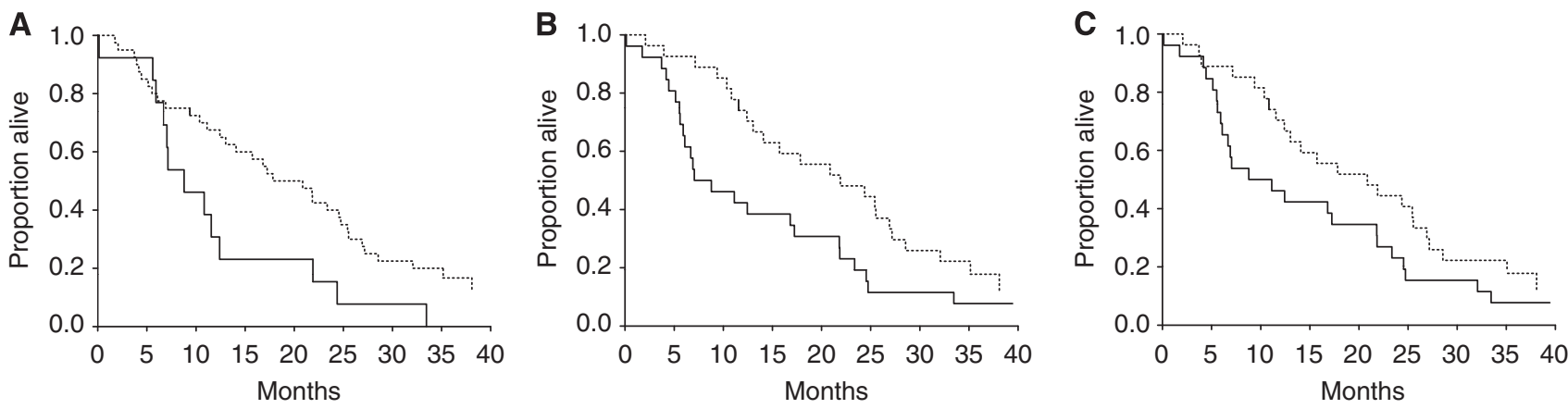

Figure 2. OS according to the level of cfDNA, MTV and TLG. (A) Dashed line: $<75 \%$, solid line: $>75 \%$, (B and C); dashed line: $<50 \%$, Solid line: > 50\%. (A) OS according to cfDNA, median OS: <75\%: 17.8 (13-24.5) and >75\%: 8.8 (6.7-11.5); HR: 2.05 (95\% Cl 0.94-4.5); $P=0.02$. (B) OS according to MTV, median OS: <50\%: 21.9 (13.0-26.9) and >50\%: 7.0 (5.9-16.8); HR: 1.87 (95\% Cl 1.04-3.38); $P=0.02$. (C) OS according to TLG, median OS: <50\%: 20.9 (12.4-25.5) and >50\%: $8.8(6.1-17.2) ; \mathrm{HR}: 1.61(95 \% \mathrm{Cl} 0.9-2.88) ; P=0.08$.

\begin{tabular}{|c|c|c|}
\hline \multirow[b]{2}{*}{ Variables } & \multicolumn{2}{|c|}{ OS } \\
\hline & Risk ratio $(95 \% \mathrm{Cl})$ & $\boldsymbol{P}$-value \\
\hline \multicolumn{3}{|c|}{ Plasma cfDNA } \\
\hline $\begin{array}{l}<75 \%^{a} \\
>75 \%\end{array}$ & $\begin{array}{c}2.0 \\
(1.0-3.9)\end{array}$ & 0.04 \\
\hline \multicolumn{3}{|l|}{ MTV } \\
\hline $\begin{array}{l}<\text { Median }^{\text {a }} \\
>\text { Median }^{2}\end{array}$ & $\begin{array}{c}7.1 \\
(0.5-98.8)\end{array}$ & 0.1 \\
\hline \multicolumn{3}{|l|}{ TLG } \\
\hline $\begin{array}{l}<\text { Median }^{\mathrm{a}} \\
>\text { Median }\end{array}$ & $\begin{array}{c}0.2 \\
(0.02-3.4)\end{array}$ & 0.3 \\
\hline \multicolumn{3}{|l|}{ PS } \\
\hline $\begin{array}{l}0-1^{\mathrm{a}} \\
2\end{array}$ & $\begin{array}{c}1.1 \\
(0.3-3.8)\end{array}$ & 0.9 \\
\hline \multicolumn{3}{|l|}{ Stage } \\
\hline $\begin{array}{l}3^{a} \\
4 \\
\end{array}$ & $\begin{array}{c}0.9 \\
(0.5-1.6)\end{array}$ & 0.7 \\
\hline \multicolumn{3}{|c|}{ 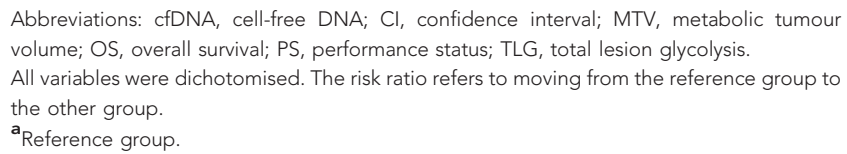 } \\
\hline
\end{tabular}

MTV and TLG, the well-documented prognostic parameters stage and PS were included. As demonstrated in Table 2, cfDNA dichotomised by the 75th percentile remained the only independent prognostic marker with a risk ratio (RR) of 2.0 (95\% CI $1.0-3.9, P=0.04)$.

\section{DISCUSSION}

New biomarkers rank high in the development of new treatment strategies in cancer. Although major efforts are made in studies of different biomarkers, many challenges remain to identify clinically reliable and hence useful biomarkers. Beyond the question of the clinical applicability, the underlying biology with its enormous molecular complexity remains obscure. Many studies in different types of cancer have demonstrated a significant prognostic value of the quantification of $\mathrm{cfDNA}$, leading to the question of whether cfDNA is merely a complicated way of measuring the tumour burden. Since the biological mechanisms behind the presence of cfDNA in the circulation remain insufficiently elucidated, the question appears to be highly relevant.

Potential sources of cfDNA are discussed by Stroun et al who find it little plausible that the primary source of cfDNA should be circulating tumour cells or micro-metastases, as the number of these is too low to provide larger amounts of cfDNA. Also, necrosis from tumour cells is discussed but is probably insufficient to entirely explain the increased levels observed with even small tumours. The most plausible sources of cfDNA are apoptosis or active release, which are mechanisms that may differ from tumour to tumour without being dependent on tumour size (Stroun et al, 2000). Another source of cfDNA may be the necrosis of normal tissue caused by the infiltrating tumour cells. This characteristic may be more pronounced in the most aggressive tumours and add explanation to the prognostic importance of cfDNA.

It is well known that cfDNA is also present in the circulation during normal conditions, probably released from normal tissue cells as well as from leukocytes and other cells undergoing lysis in the blood stream. When investigating the total amount of cfDNA in patients with malignant diseases, the risk of lysis caused by prolonged stasis during blood sampling is thus important to be aware of, as it may confound the results of the measurements. Furthermore, the risk of contaminating the plasma with leukocytes during the pipetting process should be accounted for as well as the effect of different methods in plasma separation by centrifugation may have on the final level of cfDNA in the sample. In our study, plasma was separated by centrifugation at $2000 \mathrm{~g}$ for $10 \mathrm{~min}$, and it may be discussed whether this approach is the best, or whether a second centrifugation would have improved the separation as described by Messauodi et al (2013). Also, we did not have the possibility of detecting possible leukocyte contamination, but in a previous study by Spindler et al (2012) using the same method, a high correlation between the level of cfDNA and plasma mutated, thus tumour-specific DNA was observed, supporting the reliability of our method.

There is no gold standard for evaluation of tumour burden, but PET/CT scans offer a fairly good instrument. Moreover, PET/CT provides information on the metabolic activity, which may serve as a marker of tumour aggressiveness. When estimating the tumour volume by PET/CT it is, however, important to be aware of potential sources of bias. A small number of lung tumours do not present increased FDG uptake, for example, some bronchoalveolar carcinomas, carcinoids and small lesions and their lack of activity at the $\mathrm{PET} / \mathrm{CT}$ scan may lead to an underestimation of the true tumour volume. Furthermore, to distinguish between inflamed and infected 
areas of atelectasis and tumour may be difficult conferring potential overestimation of the tumour burden. Metastases of the liver and brain may also be difficult to evaluate due to high physiological uptake in these organs. Specialist experience is therefore of utmost importance when evaluating the scans and consequently, the PET/CT scans in our study were evaluated by an experienced specialist of nuclear medicine blinded to the level of cfDNA.

There is no overall consensus as to which index is more appropriate for characterisation of tumour metabolism and tumour volume from FDG PET. Several methods of measuring MTV and TLG have been proposed. Some software use spatial derivatives to locate tumour boundaries (Lee et al 2007; Zaizen et al, 2012; Zhang et al, 2012), some use mediastinal background +2 s.d. as a reference (Chen et al, 2012), others use a cutoff at $50 \%$ of $\mathrm{SUV}_{\max }$ (Gould et al, 2001; Kim et al, 2012), and finally some use a fixed cutoff value of 2.5 SUV (Liao et al, 2012; Hyun et al, 2013) as presented in this study. The latter method has been quoted in textbooks for more than a decade (Hsu et al, 2003; Lin, 2009), is simple, and does not require reference values decreasing the inter-observer variation. It is a pure metabolic measurement without spatial delineation.

Our study is one of the first to examine the correlation between cfDNA and tumour burden in terms of PET parameters. In a pilot study by Kwee et al (2012), the correlation between cfDNA and PET parameters during chemotherapy treatment of hormonerefractory prostate cancer was described. Under the hypothesis that the level of cfDNA increases due to chemotherapy-induced tumour lysis, the level of cfDNA was correlated with the mean of all lesions SUV $_{\max }$. Thus, the metabolic activity and not the tumour burden was the target of interest. The study included eight patients, and some correlation between the levels of cfDNA, which indeed did increase after chemotherapy, and the $\mathrm{SUV}_{\max }$ was observed. Also, patients with the lowest level of pre-treatment cfDNA were those with the best treatment response. The study is interesting, but the limited number of patients makes definite conclusions precarious. Furthermore, the different design, tracer $\left({ }^{18} \mathrm{~F}\right.$-Fluorocholine) and patient group, makes a full comparison with our results difficult. We found no correlation between the PET parameters and cfDNA, neither when considered as tumour burden by MTV nor as tumour metabolic activity. There may be several explanations, including cfDNA not merely reflecting the tumour burden, but being a consequence of far more complicated biological mechanisms remaining to be fully understood. We estimated tumour activity by TLG, but did not find any correlation between the level of cfDNA and this parameter. This may be due to the fact that TLG is a combined measure of tumour volume and metabolic activity estimated by SUV. The correlation between MTV and TLG was almost perfect, making the observation of no correlations among MTV, TLG and cfDNA little surprising.

The prognostic value of cfDNA and the PET parameters has previously been reported in various studies (Lee et al, 2007; Chen et al, 2012; Kim et al, 2012; Liao et al, 2012; Zaizen et al, 2012; Zhang et al, 2012, 2013; Hyun et al, 2013). Some of the studies are pre-surgical (Kim et al, 2012; Hyun et al, 2013; Zhang et al, 2013), others are non-surgical (Liao et al, 2012; Zaizen et al, 2012), and some include both NSCLC and SCLC (Lee et al, 2007). Most studies are retrospective, and in some cases the number of patients is low. The data are thus still sparse and require confirmation in large, methodologically uniform studies. In our study, we demonstrated a significantly shorter OS of patients with either cfDNA above the 75th percentile or MTV above the median, which is in accordance with a previous study by Zhang et al (2012). When performing a multivariate analysis including both parameters, the only one remaining statistically and independently significant was cfDNA. This indication of a stronger prognostic value of cfDNA than MTV supports the possible role of cfDNA as an independent prognostic marker of clinical interest.
Even though our study is one of the first in this area and strengthened by the prospective approach and independent PET evaluation, the number of patients is limited, making definite conclusions difficult. The lack of correlation between cfDNA and tumour burden supports the argument that cfDNA is a phenomenon beyond simple tumour lysis that may reflect more complex biological mechanisms. Further studies of the biological mechanisms behind the origin of cfDNA are called for.

\section{ACKNOWLEDGEMENTS}

We thank Yvette Schandorf Sørensen for keeping track of the blood sample collection and Anita Laier Høvenhoff for technical assistance. Furthermore, our thanks go to Karin Larsen for proofreading the manuscript.

CONFLICT OF INTEREST

The authors declare no conflict of interest.

\section{REFERENCES}

Boellaard R, O'Doherty MJ, Weber WA, Mottaghy FM, Lonsdale MN, Stroobants SG, Oyen WJ, Kotzerke J, Hoekstra OS, Pruim J, Marsden PK, Tatsch K, Hoekstra CJ, Visser EP, Arends B, Verzijlbergen FJ, Zijlstra JM, Comans EF, Lammertsma AA, Paans AM, Willemsen AT, Beyer T, Bockisch A, Schaefer-Prokop C, Delbeke D, Baum RP, Chiti A, Krause BJ (2010) FDG PET and PET/CT: EANM procedure guidelines for tumour PET imaging: version 1.0. Eur J Nucl Med Mol Imaging 37: 181-200.

Chen HH, Chiu NT, Su WC, Guo HR, Lee BF (2012) Prognostic value of whole-body total lesion glycolysis at pretreatment FDG PET/CT in non-small cell lung cancer. Radiology 264: 559-566.

Dawson SJ, Tsui DW, Murtaza M, Biggs H, Rueda OM, Chin SF, Dunning MJ, Gale D, Forshew T, Mahler-Araujo B, Rajan S, Humphray S, Becq J, Halsall D, Wallis M, Bentley D, Caldas C, Rosenfeld N (2013) Analysis of circulating tumor DNA to monitor metastatic breast cancer. N Engl J Med 368: 1199-1209.

De Mattos-Arruda L, Olmos D, Tabernero J (2011) Prognostic and predictive roles for circulating biomarkers in gastrointestinal cancer. Future Oncol 7: 1385-1397.

De MM, Klatte T, Haitel A, Marberger M (2011) Serum cell-free DNA in renal cell carcinoma: a diagnostic and prognostic marker. Cancer 118: 82-90.

Ellinger J, Muller SC, Stadler TC, Jung A, RA VON, Bastian PJ (2011) The role of cell-free circulating DNA in the diagnosis and prognosis of prostate cancer. Urol Oncol 29: 124-129.

Gorenstein LA, Sonett JR (2011) The surgical management of stage I and stage II lung cancer. Surg Oncol Clin N Am 20: 701-720.

Gould MK, Maclean CC, Kuschner WG, Rydzak CE, Owens DK (2001) Accuracy of positron emission tomography for diagnosis of pulmonary nodules and mass lesions: a meta-analysis. JAMA 285: 914-924.

Hsu WH, Hsu NY, Shen YY, Yen RF, Kao CH (2003) Differentiating solitary pulmonary metastases in patients with extrapulmonary neoplasmas using FDG-PET. Cancer Invest 21: 47-52.

Hyun SH, Choi JY, Kim K, Kim J, Shim YM, Um SW, Kim H, Lee KH, Kim BT (2013) Volume-based parameters of 18F-fluorodeoxyglucose positron emission tomography/computed tomography improve outcome prediction in early-stage non-small cell lung cancer after surgical resection. Ann Surg 257: 364-370.

Kim K, Kim SJ, Kim IJ, Kim YS, Pak K, Kim H (2012) Prognostic value of volumetric parameters measured by F-18 FDG PET/CT in surgically resected non-small-cell lung cancer. Nucl Med Commun 33: 613-620.

Kwee S, Song MA, Cheng I, Loo L, Tiirikainen M (2012) Measurement of circulating cell-free DNA in relation to $18 \mathrm{~F}$-fluorocholine PET/CT imaging in chemotherapy-treated advanced prostate cancer. Clin Transl Sci 5: 65-70.

Lee P, Weerasuriya DK, Lavori PW, Quon A, Hara W, Maxim PG, Le QT, Wakelee HA, Donington JS, Graves EE, Loo Jr BW (2007) Metabolic 
tumor burden predicts for disease progression and death in lung cancer. Int J Radiat Oncol Biol Phys 69: 328-333.

Leon SA, Shapiro B, Sklaroff DM, Yaros MJ (1977) Free DNA in the serum of cancer patients and the effect of therapy. Cancer Res 37: 646-650.

Liao S, Penney BC, Wroblewski K, Zhang H, Simon CA, Kampalath R, Shih MC, Shimada N, Chen S, Salgia R, Appelbaum DE, Suzuki K, Chen CT, Pu Y (2012) Prognostic value of metabolic tumor burden on 18F-FDG PET in nonsurgical patients with non-small cell lung cancer. Eur J Nucl Med Mol Imaging 39: 27-38.

Lin EC (2009) PET and PET/CT, A Clinical Guide, 2nd edn. Thieme: New York.

McShane LM, Altman DG, Sauerbrei W, Taube SE, Gion M, Clark GM (2005) REporting recommendations for tumour MARKer prognostic studies (REMARK). Eur J Cancer 41: 1690-1696.

Messaoudi SE, Rolet F, Mouliere F, Thierry AR (2013) Circulating cell free DNA: preanalytical considerations. Clin Chim Acta 424: 222-230.

Mok TS, D'arcangelo M, Califano R (2012) Clinical outcomes with erlotinib in patients with epidermal growth factor receptor mutation. Drugs 72(Suppl 1): 3-10.

Nygaard AD (2012) Quantification of cell free DNA as a prognostic factor in advanced NSCLC. ESMO Congress 2012.

Punnoose EA, Atwal S, Liu W, Raja R, Fine BM, Hughes BG, Hicks RJ, Hampton GM, Amler LC, Pirzkall A, Lackner MR (2012) Evaluation of circulating tumor cells and circulating tumor DNA in non-small cell lung cancer: association with clinical endpoints in a phase II clinical trial of pertuzumab and erlotinib. Clin Cancer Res 18: 2391-2401.

Rinaldi M, Cauchi C, Gridelli C (2006) First line chemotherapy in advanced or metastatic NSCLC. Ann Oncol 17(Suppl 5): v64-v67.

Schwarzenbach H, Hoon DS, Pantel K (2011) Cell-free nucleic acids as biomarkers in cancer patients. Nat Rev Cancer 11: 426-437.

Spindler KL, Pallisgaard N, Vogelius I, Jakobsen A (2012) Quantitative cell-free DNA, KRAS, and BRAF mutations in plasma from patients with metastatic colorectal cancer during treatment with cetuximab and irinotecan. Clin Cancer Res 18: 1177-1185.
Steffensen KD, Waldstrom M, Grove A, Lund B, Pallisgard N, Jakobsen A (2011) Improved classification of epithelial ovarian cancer: results of 3 Danish cohorts. Int J Gynecol Cancer 9: 1592-1600.

Stroun M, Maurice P, Vasioukhin V, Lyautey J, Lederrey C, Lefort F, Rossier A, Chen XQ, Anker P (2000) The origin and mechanism of circulating DNA. Ann NY Acad Sci 906: 161-168.

Therasse P, Arbuck SG, Eisenhauer EA, Wanders J, Kaplan RS, Rubinstein L, Verweij J, Van GM, van Oosterom AT, Christian MC, Gwyther SG (2000) New guidelines to evaluate the response to treatment in solid tumors. European Organization for Research and Treatment of Cancer, National Cancer Institute of the United States, National Cancer Institute of Canada. J Natl Cancer Inst 92: 205-216.

Vadakara J, Borghaei H (2012) Personalized medicine and treatment approaches in non-small-cell lung carcinoma. Pharmgenomics Pers Med 5: 113-123.

Zaizen Y, Azuma K, Kurata S, Sadashima E, Hattori S, Sasada T, Imamura Y, Kaida H, Kawahara A, Kinoshita T, Ishibashi M, Hoshino T (2012) Prognostic significance of total lesion glycolysis in patients with advanced non-small cell lung cancer receiving chemotherapy. Eur J Radiol 81: 4179-4184.

Zhang H, Wroblewski K, Appelbaum D, Pu Y (2012) Independent prognostic value of whole-body metabolic tumor burden from FDG-PET in non-small cell lung cancer. Int J Comput Assist Radiol Surg 8: 181-191.

Zhang H, Wroblewski K, Liao S, Kampalath R, Penney BC, Zhang Y, Pu Y (2013) Prognostic value of metabolic tumor burden from (18)F-FDG PET in surgical patients with non-small-cell lung cancer. Acad Radiol 20: $32-40$.

This work is published under the standard license to publish agreement. After 12 months the work will become freely available and the license terms will switch to a Creative Commons AttributionNonCommercial-Share Alike 3.0 Unported License. 